\title{
Adipocyte differentiation influences the proliferation and migration of normal and tumoral breast epithelial cells
}

\author{
VIRGINIA PISTONE CREYDT ${ }^{1}$, PAULA ALEJANDRA SACCA ${ }^{1}$, AMELIA JULIETA TESONE ${ }^{1}$, \\ LUCIANO VIDAL ${ }^{3}$ and JUAN CARLOS CALVO ${ }^{1,2}$
}

\begin{abstract}
${ }^{1}$ Laboratorio de Proteoglicanos y Matriz Extracelular, Instituto de Biología y Medicina Experimental, Consejo Nacional de Investigaciones Científicas y Técnicas; ${ }^{2}$ Departamento de Química Biológica, Facultad de Ciencias Exactas y Naturales, Universidad de Buenos Aires, Buenos Aires, Argentina; ${ }^{3}$ Stem Center, Palma de Mallorca, Islas Baleares, Spain
\end{abstract}

Received December 14, 2009; Accepted February 16, 2010

DOI: $10.3892 / \mathrm{mmr} 00000276$

\begin{abstract}
Stromal tissue regulates the development and differentiation of breast epithelial cells, with adipocytes being the main stromal cell type. The aim of the present study was to evaluate the effect of adipocyte differentiation on proliferation and migration, as well as to assess the activity of heparanase and metalloproteinase-9 (MMP-9), in normal (NMuMG) and tumoral (LM3) murine breast epithelial cells. NMuMG and LM3 cells were grown on irradiated 3T3-L1 cells (stromal support, SS) at various degrees of differentiation [preadipocytes (preA), poorly differentiated adipocytes (pDA) and mature adipocytes (MA)] and/or were incubated in the presence of conditioned medium (CM) derived from each of these three types of differentiated cells. Cells grown on a plastic support or in fresh medium served as the controls. Cell proliferation was measured with a commercial colorimetric kit, and the motility of the epithelial cells was evaluated by means of a wound-healing assay. Heparanase activity was assessed by quantifying heparin degradation, and the expression of MMP-9 was determined using Western blotting. The results indicate that cell proliferation was increased after 24 and $48 \mathrm{~h}$ in the NMuMG and LM3 cells grown on preA, pDA and MA $\mathrm{SS}$. In the NMuMG cells cultured on SS in the presence of all three types of CM, proliferation was enhanced. LM3 cell migration was increased in the presence of all three types of $\mathrm{CM}$ and in cells grown on preA SS. Heparanase activity was increased in the NMuMG cells incubated with all three types of CM, and in the LM3 cells incubated with the CM from pDA and MA. Both the NMuMG and LM3 cell lines presented basal expression of MMP-9; however, a significant increase in MMP-9 expression was observed in the LM3 cells incubated with each of the three types of CM. In conclusion, adipocyte
\end{abstract}

Correspondence to: Dr Juan Carlos Calvo, Instituto de Biología y Medicina Experimental, Consejo Nacional de Investigaciones Científicas y Técnicas, Vuelta de Obligado 2490, Buenos Aires, Argentina

E-mail: jcalvo@dna.uba.ar

Key words: cancer, adipocyte, epithelial-stromal interactions differentiation influences normal and tumoral breast epithelial cell proliferation and migration. Heparanase and MMP-9 appear to be involved in this regulation. The experimental model presented in this study is in keeping with the characteristics of the physiological environment of breast epithelial cells, in terms of both the soluble and insoluble factors present and the stromal structure per se.

\section{Introduction}

Breast tissue changes throughout a woman's life, mainly during the periods of fertility and pregnancy. Breast epithelial cells consequently undergo constant growth, development and differentiation. The stromal compartment plays a key role in the regulation of these changes through soluble factors that are released into the environment, as well as through insoluble factors that are present in the stroma itself. Adipocytes are highly active endocrine cells that not only secrete a host of soluble factors, but also contribute very significantly to the unique make-up of the extracellular matrix (ECM) (1). In the microenvironment of mammary tissue, adipocytes are among the predominant stromal cell types (1), and are present in the form of preadipocytes, mature adipocytes and adipocytes in intermediate differentiation states (poorly differentiated adipocytes) (2). Although numerous studies have attempted to elucidate adipocyte-ECM interactions, they have yet to clarify the essential role of adipose tissue in the development and function of the mammary gland (3). The preadipocyte cell line 3T3-L1, derived from the parent 3T3 fibroblast cell line, has previously been used to study adipocyte differentiation (4-6). Until recently, adipocytes were mainly considered to be an energy storage depot; however, there is now clear evidence indicating that fat tissue is an endocrine organ that produces hormones, growth factors, adipokines and other molecules, which may affect normal duct development as well as tumor growth and metastasis (2).

An organ's function depends on the cell types of which it is composed as well as on its global organization. Loss of tissue architecture is a prerequisite for most types of cancers, while normal architecture in an organ may act as a potent tumor suppressor, preventing malignant phenotypes even in cells with significant genomic abnormalities $(7,8)$. Worldwide, breast 
cancer is currently the most common cancer affecting women (9). Tumoral progression occurs within a microsystem where cancer and stromal cells exchange proteinases, cytokines and other soluble compounds that promote cell growth. In addition to the release of these components into the environment, a reciprocal influence between the two cell types by means of direct cell to cell contact (10) and through modifications in the ECM (11) has been proposed. The invasion of healthy tissue by tumor cells is promoted by local ECM proteolysis. This indicates that the ECM, besides being a support system, plays a key role in the regulation of cell size, transport and migration, and in the expression of specific genes $(12,13)$. To date, little is known regarding the specific mechanisms by which tumor cells activate stromal tissue, or how the stroma regulates the biology of carcinogenesis (14).

Heparan sulphate (HS) is the main proteoglycan component of cell membranes and the ECM. HS chains interact through specific attachment sites with the main protein components of the basement membrane and ECM. Heparanase is an endo- $\beta$ D-glucuronidase capable of cleaving heparan sulphate on the cell surface and ECM. This activity is strongly implicated in tumor metastasis and ECM remodeling (15-17). Nevertheless, at present, the regulatory mechanisms of heparanase expression are unknown. Increased expression of heparanase mRNA and protein has been reported in a variety of metastatic cell lines and human tumoral tissue, whereas adjacent normal tissue does not exhibit detectable levels of heparanase (16).

ECM proteoglycans are cleaved by proteases. A balance between the synthesis and removal of matrix components is essential for the maintenance of ECM function. Additionally, tumor progression requires ECM degradation and tissue remodeling. These processes involve metalloproteinases (MMPs), which are members of an endopeptidase family that cleaves ECM components, as well as different cell modulators secreted by or present on the cell surface (18). Under physiological conditions, MMP activity is regulated by transcription levels, precursor activation, interaction with other ECM components and inhibition by endogenous inhibitors. Abnormal MMP activity induces various pathologies, including arthritis, fibrosis, nephritis, and tumoral growth and metastasis (18). Researchers have found that increased MMP-2 and -9 expression in human breast tumors is associated with an unfavorable patient prognosis. These two metalloproteinases are capable of degrading type IV collagen (9), which is abundant in basement membranes and which separates epithelial cells from the underlying stroma. Thus, increased expression and activity of MMP-2 and -9 in tumors leads to the degradation of the basement membrane, which is an essential step in tumor invasion.

The aim of this study was to evaluate the effect of various types of stromal support (insoluble factors) and/or conditioned media (soluble factors) obtained from distinct adipocyte differentiation states on the proliferation and migration of normal (NMuMG) and tumoral (LM3) murine breast epithelial cells. Additionally, changes in heparanase activity and MMP-9 expression were quantified in both the epithelial cell lines previously incubated with the three types of 3T3-L1 cell conditioned media, in order to evaluate the possible involvement of these proteins in the observed cell proliferation and migration.

\section{Materials and methods}

Reagents. Reagents were obtained from Sigma Chemical Co. (St. Louis, MO, USA). All tissue culture flasks, dishes and multi-well plates were from Falcon (Orange Scientific, Graignette Business Park, Belgium).

Cell lines and culture. 3T3-L1 murine preadipocytes were obtained from the American Type Culture Collection (Rockville, MD, USA). Cells were cultured in T-75 flasks in DMEM-F12 medium with $10 \%$ fetal bovine serum (FBS) in a $5 \% \mathrm{CO}_{2}$ atmosphere at $37^{\circ} \mathrm{C}$. NMuMG (also from the American Type Culture Collection) and LM3 murine breast epithelial cells (kindly provided by Dr Elisa Bal de Kier Joffé, Instituto Roffo, Buenos Aires, Argentina) were cultured under the same conditions as the 3T3-L1 cells.

Differentiation of 3T3-L1 cells. 3T3-L1 cells in three differentiation states were used for stromal support (SS), and were also used to produce the three types of conditioned medium (CM). The stages of differentiation were i) preadipocytes (preA), grown to $80 \%$ confluence; ii) poorly differentiated adipocytes (pDA), grown to $100 \%$ confluence for $48 \mathrm{~h}$ followed by posterior incubation with differentiation medium for $48 \mathrm{~h}$; and iii) mature adipocytes (MA), grown to $100 \%$ confluence for $48 \mathrm{~h}$ then treated to achieve accelerated differentiation (since 3T3-L1 cells take 10-20 days to spontaneously differentiate into MA) as follows: 2 days after reaching confluence, the cells were cultivated for $48 \mathrm{~h}$ with differentiation medium $(0.5 \mathrm{mM}$ 3-isobutyl-1-methylxanthine and $0.1 \mu \mathrm{M}$ dexamethasone) (4-6). Subsequently, fresh medium containing $2 \mathrm{mM}$ insulin was added to the culture. Within 2-3 days of this treatment, $90-100 \%$ of the cells had differentiated into adipocytes, with increasing triglyceride content and the formation of lipidic vacuoles visible under a light microscope. Cells were cultured in T-75 flasks and incubated in a $5 \% \mathrm{CO}_{2}$ atmosphere at $37^{\circ} \mathrm{C}$.

Preparation of conditioned medium. To prepare the three types of $\mathrm{CM}$, fresh medium with $1 \% \mathrm{SFB}$ was added to the 3T3-L1 cells $\left(1.5 \times 10^{6} / \mathrm{ml}\right)$ in the preA, pDA and MA states (achieved as described above), and the cells were incubated for an additional $24 \mathrm{~h}$. Subsequently, the supernatants were collected and the cells removed by centrifugation. The cell-free conditioned medium was used directly in the experiments.

Preparation of stromal support. In order to evaluate the interaction between different cell types, we previously developed a method that generates a layer of cells which attaches to the culture capsule, thus permitting modification according to the experimental requirements (20). This method consists of first plating the cells on a culture plate that serves as the 'feeder layer', or material which is attached to the substrate (substrateattached material, SAM). Once the cells reach confluence, the medium is removed and the cells are washed with PBS, then the plate is placed in a plastic bag, sealed to maintain sterility and submitted to two cycles of 2 min each of microwave oven irradiation at $650 \mathrm{~W}$. This method ensures a variation in the choice of matrices according to the requirements of the experiment. SAM was produced for the 3T3-L1 cells in each of the three differentiation states described above. 
Cell proliferation assay. To determine the extent of cell proliferation, NMuMG and LM3 cells were respectively co-cultured with the irradiated 3T3-L1 cells in the three states of differentiation (preA, pDA or MA SS) and/or in the presence of the CM derived from the preA, pDA and MA 3T3-L1 cells. Cells grown on a plastic support or in the absence of $\mathrm{CM}$ served as the controls. Following culture, both cell lines were individually plated on 96-well plates in the presence of complete DMEM-F12 medium, and cell proliferation was measured using a commercial colorimetric kit (CellTiter 96 AQueous One Solution Cell Proliferation Assay, MTS; Promega). Results were expressed as the percentage of color intensity measured using a spectrophotometer, with $100 \%$ corresponding to the value obtained for the controls.

Adhesion of cells grown on stromal support. To determine the initial adhesion of the NMuMG and LM3 cells to the stromal supports, the cell lines were individually incubated at concentrations of $1 \times 10^{5}$ and $5 \times 10^{5}$ cells $/ \mathrm{ml}$ on preA, pDA or MA SS for $30 \mathrm{~min}$ at $37^{\circ} \mathrm{C}$. Non-attached cells were then removed, fresh medium was added, and the attached cells were quantified using the MTS assay as described above. Results were expressed as the percentage of color intensity measured using a spectrophotometer, with $100 \%$ corresponding to the value obtained for the control.

Cell migration (wound-healing) as say. The effect of the preA, pDA and MA SS and of the CM from the preA, pDA and MA 3T3-L1 cells on the motility of the epithelial cell lines was evaluated by means of a wound-healing assay. In the first experiment, the NMuMG and LM3 cells were plated at $1.5 \times 10^{5}$ cells $/ \mathrm{ml}$ on the preA, pDA and MA SS or on a plastic support (as a control) in 10\% FBS medium. Once 100\% confluence was achieved, $\sim 400-\mu \mathrm{m}$-wide parallel wounds were formed in both cell lines with a plastic micropipette tip. In the second experiment, the NMuMG and LM3 cells were plated on 24-well plates and grown to $100 \%$ confluence, then $\sim 400-\mu$ m-wide parallel wounds were formed with a plastic micropipette tip. CM from the preA, pDA and MA 3T3-L1 cells or fresh medium (as a control) was then added to the epithelial cells. Images were captured with an Olympus SC35 Type 12 camera at 0 and $6 \mathrm{~h}$ after wound formation in order to determine the degree of healing. An image-analysis program (ImageJ; NIH, Bethesda, MD, USA) was used to quantify the differences, observed in comparison with the controls.

Heparanase activity assay. In order to evaluate the enzymatic activity of heparanase, heparin degradation was quantified in the NMuMG and LM3 cells incubated with CM from the preA, pDA and MA 3T3-L1 cells (21). Briefly, $15 \mu \mathrm{l}$ each of the NMuMG or LM3 cells $\left(5 \times 10^{6}\right.$ cells $\left./ \mathrm{ml}\right)$ were incubated with commercial heparin $(2 \mu \mathrm{g} / 5 \mu \mathrm{l})$ at $37^{\circ} \mathrm{C}$ for $48 \mathrm{~h}$. After incubation, $6 \mu \mathrm{l}$ of $4 \mathrm{X}$ sample buffer was added, and the samples were loaded on a non-denaturing 20\% PAGE gel and stained with Rubipy (a cationic dye). Heparin $(0.4 \mu \mathrm{g} /$ $\mu \mathrm{l}$ ) was used as the loading control. The magnitude of heparin degradation was assessed by densitometric analysis of the gels using ImageJ software. Differences were observed in comparison with the control.
MMP-9 activity assay. The expression of MMP-9 was determined using Western blotting. NMuMG and LM3 cells incubated with CM from the preA, pDA and MA 3T3-L1 cells were washed twice with ice-cold PBS and scraped in lysis buffer [50 mM Tris (pH 7.4), $250 \mathrm{mM} \mathrm{NaCl}, 5$ mM EDTA, 1\% NP40, $0.1 \%$ SDS, $0.5 \%$ sodium deoxycholate and $1 \mathrm{mM}$ phenylmethylsulphonyl fluoride] containing $1 \%$ protease inhibitor cocktail (Calbiochem). Lysates were obtained by centrifugation at $13,000 \mathrm{rpm}$ for $10 \mathrm{~min}$ at $4^{\circ} \mathrm{C}$, and protein concentration was determined using the Bradford Protein Assay kit (Pierce, Rockford, IL, USA). Total protein (20-40 $\mu \mathrm{g})$ was subjected to SDS-PAGE, transferred to a Hybond-C extra nitrocellulose membrane (Amersham Biosciences, Little Chalfont, UK) and probed with anti-MMP-9 (H-129) primary antibodies (Santa Cruz Biotechnology, Inc., Santa Cruz, CA, USA), followed by HRP-conjugated secondary antibodies. MMP-9 protein was detected by enhanced chemiluminescence (Amersham Biosciences). Experiments were repeated in triplicate. $\beta$-actin protein (H-19) (Santa Cruz Biotechnology, Inc.) was used as the internal control.

Statistical analysis. Data were expressed as the means \pm SEM. Statistical significance between two mean values obtained from different experimental conditions was determined using the Student's t-test. The criterion for significance was $\mathrm{P}<0.05$.

\section{Results}

Proliferation of NMUMG and LM3 cells grown on stromal support and/or incubated with conditioned medium. The effect of adipocyte differentiation on the growth of NMuMG and LM3 cells cultured on preA, pDA or MA stromal support and/or with conditioned media from the preA, pDA or MA 3T3-L1 cells was first evaluated. After 24 and $48 \mathrm{~h}$ of incubation, a significant increase in cell proliferation was obseved in the NMuMG cells grown on the three types of SS or with the three CM, with the exception of cells grown with CM from preA after a 24-h incubation (Fig. 1A-C). The increase in cell proliferation was significantly higher in cells grown on pDA or MA SS $(\mathrm{P}<0.001)$, compared to cells incubated in the presence of $\mathrm{CM}$ from $\mathrm{pDA}$ or MA $(\mathrm{P}<0.05)$ (Fig. 1B and C). Moreover, after $48 \mathrm{~h}$ of incubation, enhanced proliferation was observed in the NMuMG cells grown on preA, $\mathrm{pDA}$ and MA SS in the presence of each of the three types of CM, in comparison to cells grown on SS in the absence of CM. These results indicate that soluble factors (CM) as well as insoluble factors (SS) produced by the adipocytes stimulated NMuMG cell growth. LM3 cell proliferation was significantly increased in cells grown on preA, pDA and MA SS for 24 or $48 \mathrm{~h}$. However, no change in growth was observed in the presence of the conditioned media (Fig. 1A-C). This indicates that the growth of LM3 cells was only stimulated by insoluble factors present in the three types of stromal support.

Adhesion of NMuMG and LM3 cells grown on stromal support. In order to verify that the increased proliferation observed in the epithelial cell lines grown on preA, pDA and MA SS was not due to an increase in the initial adhesion of the cells to the supports, we evaluated the adhesion of two 
A

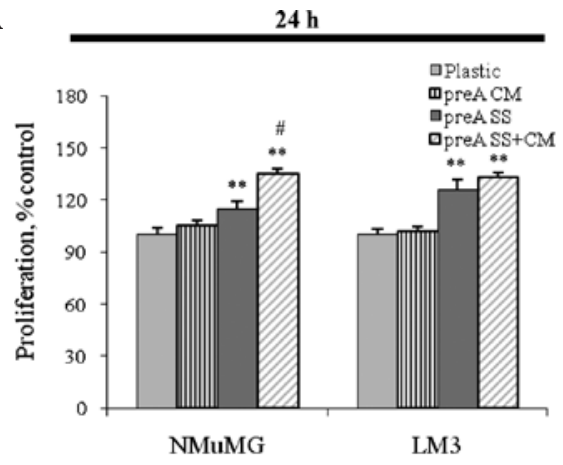

B

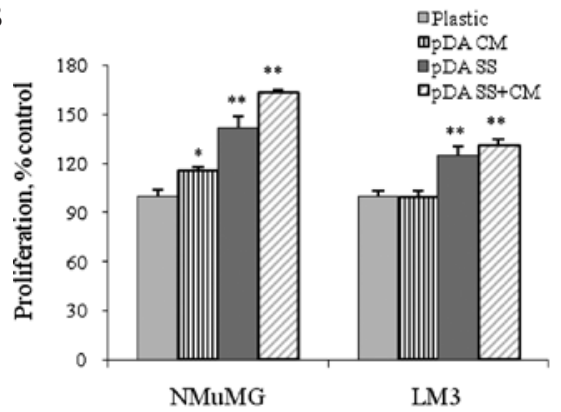

C

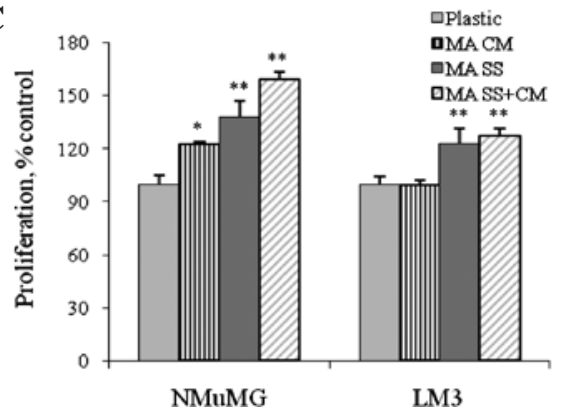

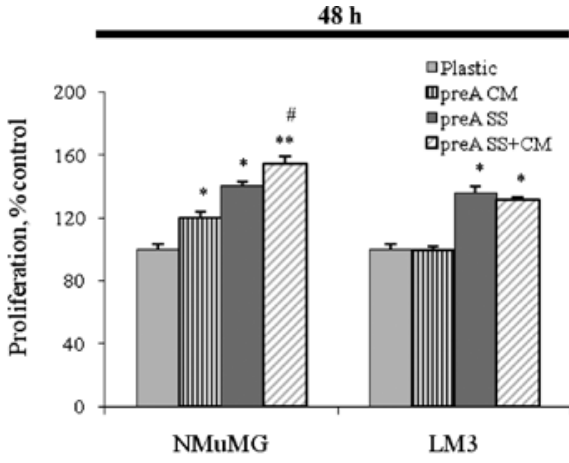
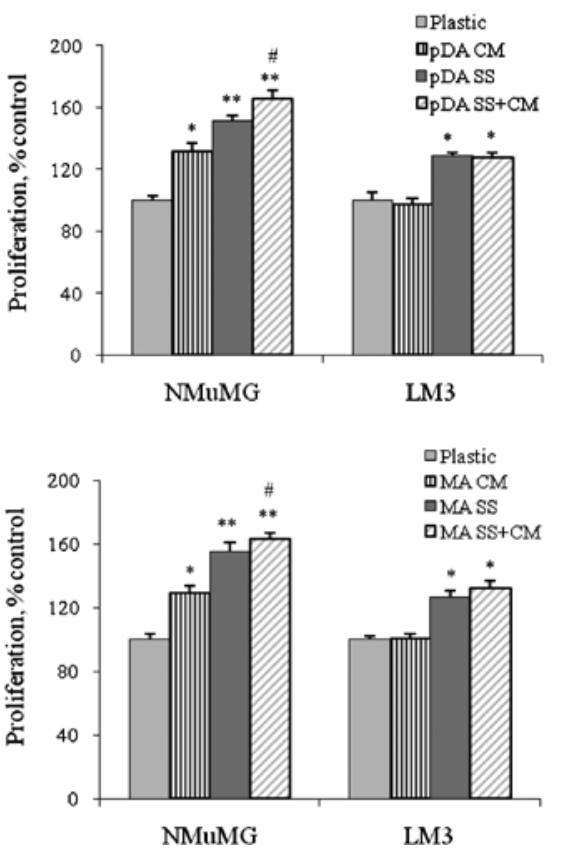

Figure 1. Effect of conditioned medium (CM) and/or stromal support (SS) on the proliferation of epithelial NMuMG and LM3 cells. Epithelial cells were grown on preA (A), pDA (B) or MA (C) 3T3-L1 irradiated cells or on plastic, in the presence or absence of CM, for 24 and 48 h. Cell proliferation was quantified using the MTS assay. Data are shown as the means \pm SEM ( $\mathrm{n}=5-6$, each experiment performed in five replicates). ${ }^{*} \mathrm{P}<0.05$ or ${ }^{* *} \mathrm{P}<0.001$ compared to growth on plastic or with fresh medium, respecively; ${ }^{\mathrm{P}}<0.05$ compared to growth on SS without CM.

A

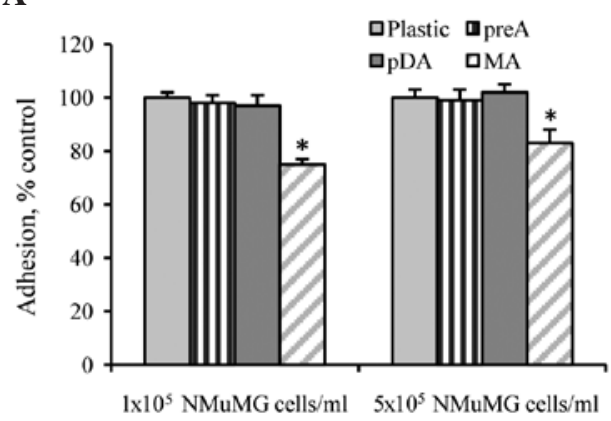

B

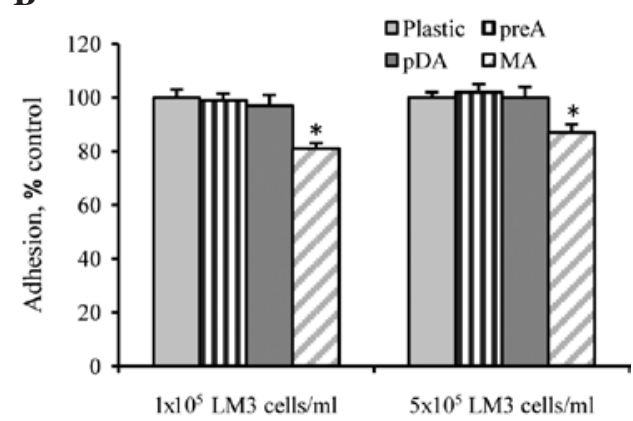

Figure 2. Adhesion of NMuMG (A) and LM3 (B) cells grown on preA, pDA or MA 3T3-L1 stromal support (SS) cells. Epithelial cells (1x105 or 5x105/ml) were grown on preA, pDA or MA SS. After $30 \mathrm{~min}$, cell attachment was quantified using the MTS assay. Data are shown as the means \pm SEM (n=3, each experiment performed in five replicates). ${ }^{*} \mathrm{P}<0.05$ compared to growth on plastic (control).

different concentrations of NMuMG and LM3 cells after $30 \mathrm{~min}$ of contact with the stromal supports compared to the plastic support. Fig. 2 shows that initial cell adhesion was not increased in the epithelial cells grown on the stromal supports compared to the plastic. On the contrary, adhesion was signifi- cantly lower in the cells grown on MA SS. Therefore, the observed increase in the proliferation of the cell lines grown on all three types of stromal support was due to a stimulation of the substrate-attached material per se, and not to an increased initial adhesion to the substrates. 
A

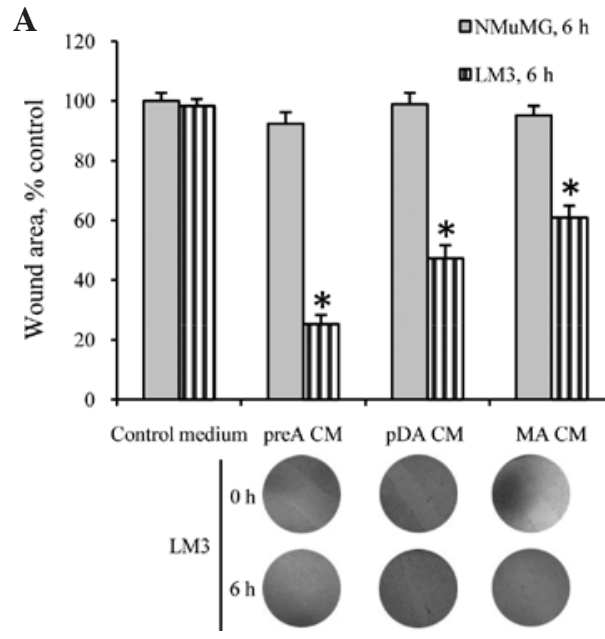

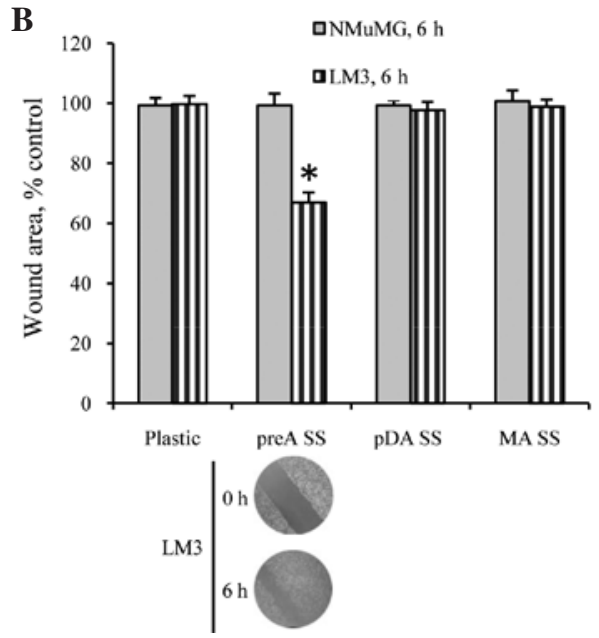

Figure 3. Effect of conditioned medium (CM) (A) or stromal support (SS) (B) on the migration of NMuMG and LM3 cells. Epithelial cells were grown to confluence on preA, pDA or MA 3T3-L1 SS (B) or on plastic (B, control) with complete DMEM-F12 medium. Wounds (400 $\mu$ m-wide) were formed, and complete DMEM-F12 medium (A, control) or CM (A) was added. Images were captured at 0 and $6 \mathrm{~h}$ after wounding. In order to quantify the differences observed in the area of the wounds, an image analysis program was used (ImageJ). ${ }^{*} \mathrm{P}<0.05$ compared to baseline. Inserts: Images captured at 0 and $6 \mathrm{~h}$ after wounding (magnification x400). (A) LM3 incubated with preA CM (column 1), pDA CM (column 2) and MA CM (column 3). (B) LM3 incubated with preA SS (column 1).
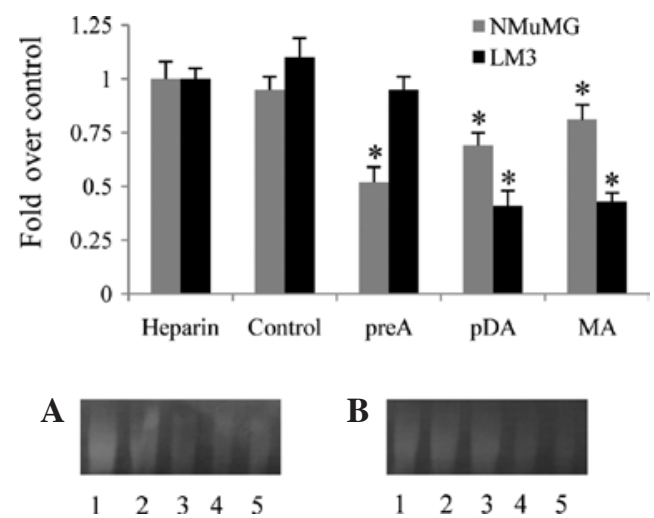

Figure 4. Heparanase activity in NMuMG or LM3 cells incubated with conditioned medium (CM) from preA, pDA or MA 3T3-L1 cells. Commercial heparin was incubated for $24 \mathrm{~h}$ with NMuMG or LM3 cells that had previously been incubated for $48 \mathrm{~h}$ in the presence of each of the three types of CM. Samples were subjected to electrophoresis, and gels were stained with Rubipy. Images were analyzed by densitometry. Data were normalized with respect to heparin without epithelial cells. Histograms represent the means \pm SEM of two independent experiments. ${ }^{*} \mathrm{P}<0.05$ compared to baseline. Insert: Images of a non-denaturing gel stained with Rubipy. (A) Heparin (control, lane 1), NMuMG + fresh medium with heparin (lane 2), NMuMG + preA CM with heparin (lane 3), NMuMG + pDA CM with heparin (lane 4), NMuMG + MA CM with heparin (lane 4). (B) As in A, but with LM3 cells.

Migration of NMUMG and LM3 cells grown on stromal support or incubated with conditioned medium. The motility of the NMuMG and LM3 cells grown on preA, pDA and MA SS or in the presence of CM from preA, pDA and MA was next evaluated. After wounding with a pipette tip, the NMuMG cells showed no significant differences in migration (wound-healing) $6 \mathrm{~h}$ after the initial wounding under any of the assay conditions (Fig. 3A and B). By contrast, LM3 migration was increased in the epithelial cells grown with all three types of $\mathrm{CM}$, as wound healing occurred in $75 \pm 3 \%$ (preA), $53 \pm 4 \%(\mathrm{pDA})$ and $39 \pm 4 \%$ (MA) of cells after a $6-\mathrm{h}$ incubation $(\mathrm{P}<0.05)$ (Fig. 3A). In LM3 cells cultured on
NMuMG
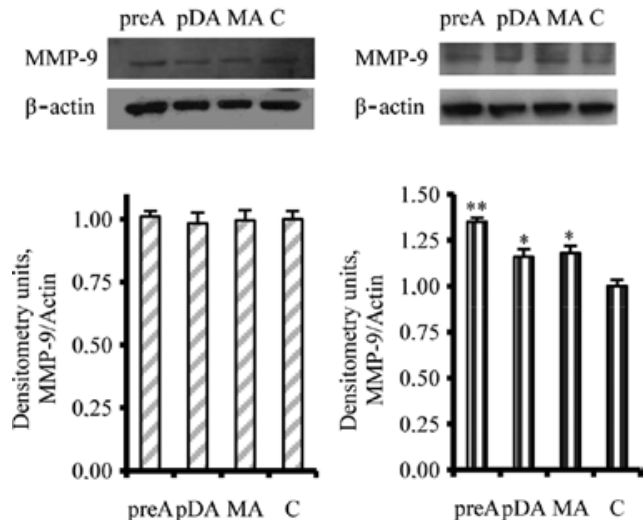

Figure 5. Expression of MMP-9 in NMuMG or LM3 cells incubated with conditioned medium from preA, pDA or MA 3T3-L1 cells, or with fresh medium as a control (C). Samples were subjected to SDS-PAGE with a primary antibody specific for MMP-9. $\beta$-actin protein was used as the internal control. Images were analyzed by densitometry. Histograms represent the means \pm SEM of three independent experiments. ${ }^{*} \mathrm{P}<0.05$ and ${ }^{* *} \mathrm{P}<0.01$ compared to basal levels of MMP-9.

stromal support, migration was observed only in cells grown on the preA SS $(33 \pm 3 \%)(\mathrm{P}<0.05)$, with respect to baseline conditions (Fig. 3B).

Heparanase enzymatic activity of NMUMG and LM3 cells incubated with conditioned medium. In order to evaluate the effect of heparanase on NMuMG and LM3 cell proliferation and migration, heparin degradation was assessed in the two cell lines previously incubated with CM from preA, pDA and MA. Fig. 4 shows a significant decrease in heparin band intensity in the NMuMG cells incubated with the three types of $\mathrm{CM}$, which corresponds to an increase in heparanase activity. By contrast, LM3 cells incubated with the three types of CM exhibited enzymatic activity. None of the cell lines presented basal heparanase activity (Fig. 4). 
Expression of MMP-9 in NMUMG and LM3 cells incubated with conditioned medium. The expression of MMP-9 in NMuMG and LM3 cells incubated with CM from preA, pDA and MA was evaluated. Both cell lines presented basal expression of MMP-9. This basal expression was not altered in NMuMG cells previously incubated with the three types of CM (Fig. 5). By contrast, a significant increase in MMP-9 expression was observed in the LM3 cells previously incubated with the conditioned media, the highest value being in LM3 cells incubated in the presence of CM from preA (Fig. 5).

\section{Discussion}

Breast epithelial cells undergo constant development and differentiation throughout a woman's life. Stromal tissue plays a key role in the regulation of these changes, as well as in the regulation of breast cancer cell growth. Preadipocytes, mature adipocytes and those in intermediate differentiation states are the main stromal cell types in breast tissue. In this study, we demonstrated that the state of adipocyte differentiation influences normal and tumoral breast epithelial cell proliferation and migration. Heparanase and MMP-9 are potentially involved in this regulation (22).

The proliferation of NMuMG cells was significantly increased in cells incubated with the three types of conditioned media, as well as in cells grown on the preA, pDA and MA 3T3-L1 stromal support cells. Moreover, the increase in NMuMG cell proliferation was enhanced when cells were grown on SS in the presence of CM. Therefore, both soluble factors (CM) and insoluble factors (SS) from adipocytes stimulated the growth of the NMuMG cells, although the effect of insoluble factors was apparently significantly greater.

In addition, the results indicate that $\mathrm{CM}$ derived from the preA, pDA and MA 3T3-L1 cells stimulated heparanase activity in the NMuMG cells. Therefore, the observed increase in NMuMG cell proliferation in cells incubated with CM may at least in part be due to the stimulation of this enzyme's activity. An increase in heparanase activity has previously been described in relation to an increase in the metastatic activity of tumor cells (8); however, our findings indicate that heparanase may also be involved in the proliferation of normal breast epithelial cells (22). In terms of MMP-9 activity, basal expression was observed in the NMuMG cells, and was not modified following incubation with any of the three types of CM.

LM3 proliferation was significantly increased in the epithelial cells grown on the preA, pDA and MA SS. No changes were observed in cells incubated in the presence of any of the three types of CM. Thus, it is likely that insoluble factors present in the preA, pDA and MA SS stimulated the proliferation of tumor cells. Chamras et al (23) demonstrated that proliferating preadipocytes, but not mature adipocytes, stimulate breast cancer cell growth, while we observed an increase in tumoral cell growth in cells grown on preA, pDA and MA SS. These differences may be due to the different types of stromal support employed in the studies. Whereas Chamras et al co-cultivated stroma with epithelial cell lines, we studied the epithelial stromal interaction by seeding the epithelial cells on natural ECM obtained through the microwave-irradiation of stromal cells.
In terms of LM3 cell migration (wound healing), migration was significantly increased in the presence of all three types of $\mathrm{CM}$, with the highest values obtained for CM from preA, as compared to $\mathrm{CM}$ from $\mathrm{pDA}$ and MA. However, in LM3 cells cultured on preA, pDA and MA SS, migration was observed only with the preA SS. LM3 cells did not exhibit basal heparanase activity; however, when the cells were incubated with CM from pDA and MA, enzymatic activity was detected. By contrast, LM3 cells presented basal expression of MMP-9, and this expression increased significantly when the cells were incubated with the three types of CM. This significant increase was particularly notable in the presence of $\mathrm{CM}$ from preA. Therefore, one or more soluble factors present in $\mathrm{CM}$ from $\mathrm{pDA}$ and MA may stimulate heparanase activity and MMP-9 expression in LM3 cells, thus increasing the migration of these cells, while MMP-9, but not heparanase, is involved in the increase in LM3 cell migration in cells incubated with $\mathrm{CM}$ from preA.

In conclusion, our results demonstrate that adipocyte differentiation influences normal and tumoral breast epithelial cell proliferation and migration. Heparanase and MMP-9 apprear to be involved in this regulation. These results broaden the view of cancer, which is at present focused on oncogenes and tumor suppressor genes, to include the viewpoint that the tumoral microenvironment co-evolves and interacts in a dynamic and reciprocal way with mutated epithelium $(24,25)$. The experimental model presented is in keeping with the characteristics of the physiological environment of breast epithelial cells in terms of both the soluble and insoluble factors present and the stromal structure per se. Further research is required to identify the stimulatory factors that play a role in the paracrine interaction between normal or tumoral breast cancer cells and the surrounding stroma. The identification of these factors may provide novel strategies for the regulation of the development of normal breast tissue and/or for the prevention and treatment of breast cancer.

\section{Acknowledgements}

This study was supported in part by grants to Dr J. Carlos Calvo from Conicet (PIP 5402) and ANPCYT (PICT 2005 no. 35084).

\section{References}

1. Iyengar P, Espina V, Williams TW, et al: Adipocyte-derived collagen VI affects early mammary tumor progression in vivo, demonstrating a critical interaction in the tumor/stroma microenvironment. J Clin Invest 115: 1163-1176, 2005.

2. Celis JE, Moreira JM, Cabezon T, Gromov P, Friis E, Rank F and Gromova I: Identification of extracellular and intracellular signaling components of the mammary adipose tissue and its interstitial fluid in high risk breast cancer patients: toward dissecting the molecular circuitry of epithelial-adipocyte stromal cell interactions. Mol Cell Proteomics 4: 492-522, 2005.

3. Couldrey C, Moitra J, Vinson C, Anver M, Nagashima K and Green J: Adipose tissue: a vital in vivo role in mammary gland development but not differentiation. Dev Dyn 223: 459-468, 2002.

4. Calvo JC, Gandjbakhche AH, Nossal R, Hascall VC and Yanagishita M: Rheological effects of the presence of hyaluronic acid in the extracellular media of differentiated 3T3-L1 preadipocyte cultures. Arch Biochem Biophys 302: 468-475, 1993.

5. Calvo JC, Rodbard D, Katki A, Chernick S and Yanagishita M: Differentiation of 3T3-L1 preadipocytes with 3-isobutyl-1methylxanthine and dexamethasone stimulates cell-associated and soluble chondroitin 4-sulfate proteoglycans. J Biol Chem 266: 11237-11244, 1991. 
6. Zizola CF, Julianelli V, Bertolesi G, Yanagishita M and Calvo JC: Role of versican and hyaluronan in the differentiation of 3T3-L1 cells into preadipocytes and mature adipocytes. Matrix Biol 26 419-430, 2007.

7. Kirshner J, Chen CJ, Liu P, Huang J and Shively JE: CEACAM1-4S, a cell-cell adhesion molecule, mediates apoptosis and reverts mammary carcinoma cells to a norma morphogenic phenotype in a 3D culture. Proc Natl Acad Sci USA 100: 521-526, 2003.

8. Weaver VM, Howlett AR, Langton-Webster B, Petersen OW and Bissell MJ: The development of a functionally relevant cell culture model of progressive human breast cancer. Semin Cancer Biol 6: 175-184, 1995.

9. Kohrmann A, Kammerer U, Kapp M, Dietl J and Anacker J: Expression of matrix metalloproteinases (MMPs) in primary human breast cancer and breast cancer cell lines: new findings and review of the literature. BMC Cancer 9: 188, 2009.

10. Zidar N, Gale N, Kambic V and Fischinger J: Proliferation of myofibroblasts in the stroma of epithelial hyperplastic lesions and squamous carcinoma of the larynx. Oncology 62: 381-385, 2002.

11. Nelson CM and Bissell MJ: Of extracellular matrix, scaffolds and signaling: tissue architecture regulates development, homeostasis and cancer. Annu Rev Cell Dev Biol 22: 287-309, 2006.

12. Bissell MJ and Radisky D: Putting tumours in context. Nat Rev Cancer 1: 46-54, 2001.

13. Reichardt LF: Extracellular matrix molecules and their receptors. In: Guidebook to the Extracellular Matrix and Adhesion Proteins. Kreiss T and Vale R (eds). Oxford University Press, New York, 1993

14. Mukaratirwa S, Koninkx JF, Gruys E and Nederbragt H: Mutual paracrine effects of colorectal tumour cells and stromal cells: modulation of tumour and stromal cell differentiation and extracellular matrix component production in culture. Int J Exp Pathol 86: 219-229, 2005

15. Cohen I, Pappo O, Elkin M, San T, Bar-Shavit R, Hazan R, Peretz T, Vlodavsky I and Abramovitch R: Heparanase promotes growth, angiogenesis and survival of primary breast tumors. Int J Cancer 118: 1609-1617, 2006
16. Hong X, Jiang F, Kalkanis SN, Zhang ZG, Zhang X, Zheng X, Jiang $\mathrm{H}$, Mikkelsen $\mathrm{T}$ and Chopp M: Increased chemotactic migration and growth in heparanase-overexpressing human U251n glioma cells. J Exp Clin Cancer Res 27: 23, 2008.

17. Nadir Y, Vlodavsky I and Brenner B: Heparanase, tissue factor and cancer. Semin Thromb Hemost 34: 187-194, 2008.

18. Egeblad M and Werb Z: New functions for the matrix metalloproteinases in cancer progression. Nat Rev Cancer 2: 161-174, 2002.

19. Visse $\mathrm{R}$ and Nagase H: Matrix metalloproteinases and tissue inhibitors of metalloproteinases: structure, function and biochemistry. Circ Res 92: 827-839, 2003.

20. Sacca PA, Pistone Creydt V, Tesone AJ and Calvo JC: A Novel and alternative in vitro method using microwave to study the epithelial-stromal interactions. Sci Res Essay (In press).

21. Rozenberg GI, Espada J, de Cidre LL, Eijan AM, Calvo JC and Bertolesi GE: Heparan sulfate, heparin and heparinase activity detection on polyacrylamide gel electrophoresis using the fluorochrome tris(2,2'-bipyridine) ruthenium (II). Electrophoresis 22: 3-11, 2001.

22. Brun R, Naroditsky I, Waterman M, Ben Izhak O, Groisman G, Ilan N and Vlodavsky I: Heparanase expression by Barrett's epithelium and during esophageal carcinoma progression. Mod Pathol 22: 1548-1554, 2009.

23. Chamras H, Bagga D, Elstner E, Setoodeh K, Koeffler HP and Heber D: Preadipocytes stimulate breast cancer cell growth. Nutr Cancer 32: 59-63, 1998.

24. Casey T, Bond J, Tighe S, Hunter T, Lintault L, Patel O, Eneman J, Crocker A, White J, Tessitore J, Stanley M, Harlow S, Weaver D, Muss H and Plaut K: Molecular signatures suggest a major role for stromal cells in development of invasive breast cancer. Breast Cancer Res Treat 114: 47-62, 2009.

25. Trimboli AJ, Cantemir-Stone CZ, Li F, et al: Pten in stromal fibroblasts suppresses mammary epithelial tumours. Nature 461: 1084-1091, 2009 